\title{
Study on the Inductance of Micro Planar Coil for Eddy Current Detection
}

\author{
Yude Wu \\ Institute of MEMS Technology \\ Beijing University of Technology \\ Beijing, China, 0086-15201226026 \\ yude_wu@emails.bjut.edu.cn
}

\author{
Bendong Liu \\ Institute of MEMS Technology \\ Beijing University of Technology \\ Beijing, China, 010-67396187 \\ liubendong@bjut.edu.cn
}

\author{
Desheng Li \\ Institute of MEMS Technology \\ Beijing University of Technology \\ Beijing, China, 010-67396993 \\ dsli@bjut.edu.cn
}

\begin{abstract}
A noncontact inductive sensor was designed for the eddy current detection, the main structure of the inductive sensor is single-layer micro-planar coil. Two groups of micro-planar coils with different wire width, wire distance and wire thickness are fabricated by using electrochemical and MEMS technology in order to make the detection. We establish the inductance and impedance mathematic models to describe the near-field eddy current effect of the micro-planar coil according to the structure of the coil. When alternate current in certain frequency circulates in the micro-planar coil, alternate magnetic field will generate around the coil. The effect of eddy current can be mainly reflected by the signal of inductance. This paper describes the fabrication of the micro-planar coil, and proposes an approach to calculate the inductance. Through the experiment of testing the inductance and impedance of the coil by using Agilent 4294A Precision Impedance Analyzer and Solartron Electro-chemical Impedance System, we make a comparison between the inductance and impedance. The experimental results indicate the signal of the inductance and impedance of the micro-planar coil can be well used in the study of eddy current field while in a certain condition, but the inductance has good sensitivity and linearity and high interference immunity. The comparison between the inductance and impedance reveals that the experimental results and the theoretical calculation results are almost consistent, and the deviation is between $3 \%$ and $12 \%$. So the mathematical model of the inductance has shown to be effective, and the intensity of the eddy current field around the coil can be better expressed by the signal of the inductance while it is more stable and sensitive.
\end{abstract}

Keywords-Micro planar coil; Eddy current; Inductance; Impedance

\section{INTRODUCTION}

With the development of MEMS in aerospace, automotive, communication engineering and many other fields, and the characteristics of small size, light weight and fast response, micro-inductive sensor has a tremendous prospect in the study of eddy current nondestructive detection. The eddy current nondestructive detection is a superior non-contacting and quick approach ${ }^{[1]}$. Furthermore, the micro-planar coil has become the novel sensitive device in the detection of the nearfield eddy current, while it has good sensitivity and linearity and high interference immunity. So it has been widely used in

Project of National Natural Science Foundation of China No.51105011; Project of Beijing Board of Education No.KM201210005015. the displacement and angle measure, nondestructive testing and bio-medical detection ${ }^{2]}$.

However, due to the limitation of the fabrication condition, structure of the coil and other factors, the micro-planar coil generally has a small inductance value and a high resistance value $^{[3]}$. So it is difficult to detect and extract the signal. Researchers change the shape of the coil and the fabrication process to raise the inductance value. However, this approach has little help to the inductance and impedance detection. On the other hand, this improved coil is not stable in the detection.

In general, micro-planar coil can be divided into planar spiral coil, planar meandering coil and three-dimensional spiral coil. The three-dimensional spiral coil and the planar meandering coil are rarely used in the experiment because of its complex structure and its high cost if it is fabricated by MEMS technology ${ }^{[4]}$. On the contrary, planar spiral coil is always a better choice in the experiment while it has a rule structure, such as square, hexagonal, octagonal and round ${ }^{[5]}$.

Micro-planar coils with different wire width, wire distance and wire thickness are used in the experiment based on the above statements. This article describes how to establish the mathematical model of the inductance, and proposes an approach to calculate the self-inductance and mutualinductance. Then make a comparison between the inductance and the impedance by testing the inductance and impedance of the micro-planar coil in different conditions. The results show that in a certain condition, the intensity of the eddy current field around the coil can be better expressed by the signal of the inductance while it is more stable and sensitive.

\section{FABRICATION AND STRUCTURE OF MICRO-PLANAR COIL}

The micro-planar coil is fabricated by a MEMS-based glass micromachining process including sputtering, photolithography, electroplating and etching. Glass is always used in the process because it can avoid the influence of electromagnetic induction caused by the silicon substrate, while it has good structural properties in the MEMS micromachining process. Because of the compatibility of the fabrication process, square micro-planar coil is often used in MEMS. The magnetic field of square micro-planar coil mainly concentrated in the center of the coil, and the turn numbers of 
the coil are always more than 20 . The main fabrication process is depicted in Fig.1. The prototype of the micro-planar coil is shown in Fig.2.



(b) PI isolation layer

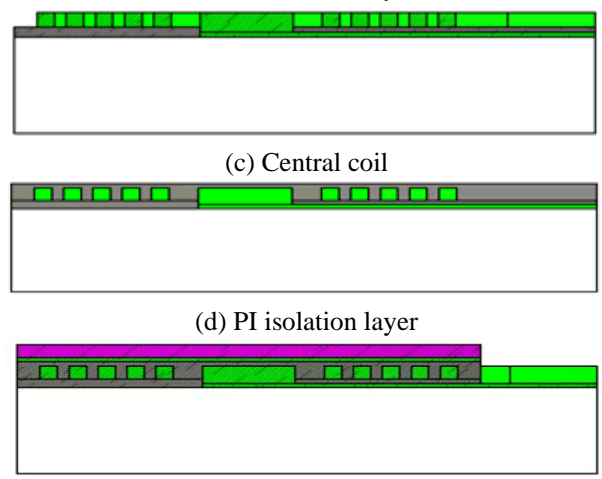

(e) Protecting plate

Figure 1. Fabrication process



Figure 2. Final structure of micro-planar coil

\section{INDUCTANCE CALCULATION OF MICRO-PLANAR COIL}

\section{A. Model analysis of micro-planar coil}

When calculate the inductance of the coil, the spirality of the coil is always ignored. Studies show the deviation caused by this simplified approach is very small ${ }^{[6]}$. Segmentation method is a relative simple method to build the mathematical model of the micro-planar coil when it is in a complex shape. In a word, the method is to divide the complex circuit of the coil into several separate segments, so that it is more convenient to calculate the inductance of each segment. If the coil consists of several straight circuits, segmentation method is more advantageous.

Assume that the magnetic permeability of medium is unrelated to the magnetic intensity, and then magnetic flux is proportional to the current. Therefore, the inductance is also unrelated to the current, but only depending on the shape of the coil, and the magnetic permeability between the conductor and the surrounding medium. Moreover, mutual-inductance is supposed to relate to the position of the wires. If the coil is made of non-magnetic material and placed in the air, the conductor can be considered to have a same magnetic permeability with the surrounding medium, and it is equal to the vacuum permeability $\mu_{0}$.

The rectangular current-carrying coil can be seen as several straight wires when the micro-planar coil is placed in the cartesian coordinate system. It is determined by the following parameters, turn number $N$, wire width $a$, wire thickness $h$ and wire distance $b$. The simplified model of the micro-planar coil is shown in Fig.3.



Figure 3. Simplified model of micro-planar coil

The mathematical model of self-inductance and mutualinductance can be expressed as (1) and (2),

$$
\begin{aligned}
L_{k} & =\frac{1}{\lambda_{k}^{2}} \int_{\lambda_{k}} d \lambda^{\prime} \int_{\lambda_{k}} \bar{M}_{k} d \lambda^{\prime \prime} \\
M_{k i} & =\frac{1}{\lambda_{k} \lambda_{i}} \int_{\lambda_{k}} d \lambda^{\prime} \int_{\lambda_{i}} \bar{M}_{k i} d \lambda^{\prime \prime} \\
\bar{M} & =\frac{\mu_{0}}{4 \pi} \int_{l^{\prime}} \int_{l^{\prime}} \frac{d l^{\prime} d l^{\prime \prime} \cos \theta}{D}
\end{aligned}
$$

Where, $l$ ' and $l$ "' are the length of the wires, $D$ is the distance between $l^{\prime}$ and $l$ ', $\theta$ is the angle between $l$ ' and $l$ ', $\lambda$ is the perimeter of wire cross-section.

\section{B. Self-inductance of the micro-planar coil}

As is shown in Fig.3, the current $i$ flows from $A$ to $B$. Set $A B=2 l_{1}, \quad B C=2 l_{2}, \quad$ then $C D=2 l_{1}+t, \quad D E=2 l_{2}+t, \quad t=a+b$. If $A B=B C=2 l$, then $C D=D E=2 l+t$. The self-inductance value can be calculated according to (1), (2) and (3).

Under the condition of direct current or alternate current in low frequency, the self-inductance value of the $n$ turn of the coil is, 


$$
\begin{aligned}
L_{n}= & \frac{\mu_{0}[2 l+2(n-1) t]}{\pi}\left[\ln \frac{2[2 l+2(n-1) t]}{a+h}+\frac{1}{2}\right]+ \\
& \frac{\mu_{0}[2 l+(2 n-1) t]}{\pi}\left[\ln \frac{2[2 l+(2 n-1) t]}{a+h}+\frac{1}{2}\right]
\end{aligned}
$$

Under the condition of alternate current in high frequency, the self-inductance value of the $n$ turn of the coil is,

$$
\begin{aligned}
L_{n}= & \frac{\mu_{0}[2 l+2(n-1) t]}{\pi}\left[\ln \frac{2[2 l+2(n-1) t]}{g}-1\right]+ \\
& \frac{\mu_{0}[2 l+(2 n-1) t]}{\pi}\left[\ln \frac{2[2 l+(2 n-1) t]}{g}-1\right]
\end{aligned}
$$

Where, $g$ is the geometric mean distance of perimeter of the rectangular section in (5).

\section{Mutual-inductance of the micro-planar coil}

When the $n$ turn of the coil is on $x$-ortho axis, the mutualinductance value between the $m$ turn and the $n$ turn of the coil is,

$$
\begin{aligned}
M_{m: n}= & \frac{\mu_{0} A}{2 \pi}\left[\ln \frac{A+\sqrt{A^{2}+F^{2}}}{F}-\frac{\sqrt{A^{2}+F^{2}}}{A}+\frac{F}{A}\right]- \\
& \frac{\mu_{0} C}{2 \pi}\left[\ln \frac{C+\sqrt{C^{2}+F^{2}}}{F}-\frac{\sqrt{C^{2}+F^{2}}}{C}+\frac{F}{C}\right]
\end{aligned}
$$

Where, $n>m$, and $A=2 l+2(m-1) t+(n-m) \mathrm{t}, C=F=(n-m) t$.

When the $n$ turn of the coil is on $x$-negative axis, the mutual-inductance value between the $m$ turn and the $n$ turn of the coil is,

$$
\begin{aligned}
M_{m: n}^{\prime}= & \frac{\mu_{0} A}{4 \pi}\left[\ln \frac{A+\sqrt{A^{2}+E^{2}}}{E}-\frac{\sqrt{A^{2}+E^{2}}}{A}+\frac{E}{A}\right]+ \\
& \frac{\mu_{0} B}{4 \pi}\left[\ln \frac{B+\sqrt{B^{2}+E^{2}}}{E}-\frac{\sqrt{B^{2}+E^{2}}}{B}+\frac{E}{B}\right]- \\
& \frac{\mu_{0} C}{4 \pi}\left[\ln \frac{C+\sqrt{C^{2}+E^{2}}}{E}-\frac{\sqrt{C^{2}+E^{2}}}{C}+\frac{E}{C}\right]- \\
& \frac{\mu_{0} D}{4 \pi}\left[\ln \frac{D+\sqrt{D^{2}+E^{2}}}{E}-\frac{\sqrt{D^{2}+E^{2}}}{D}+\frac{E}{D}\right]
\end{aligned}
$$

Where, $n>m$, and $A=2 l+(n-m) t, B=2 l+(n-m+1) t, C=(n-m) t$, $D=(n-m+1) t, E=2 l+(n-1) t+(m-1) \mathrm{t}$.

In summary, the total inductance of the micro-planar coil can be expressed as,

$$
G=\sum_{j=1}^{n} L_{j}+4 \sum_{k=1}^{m} \sum_{j=k+1}^{n} M_{m: n}+4 \sum_{k=1}^{m} \sum_{j=k}^{n} M_{m: n}^{\prime}
$$

\section{EXPERIMENTAL TEST OF THE MICRO-PLANAR COIL}

\section{A. Experimental test of the inductance model}

In order to verify the inductance models, we use Agilent 4294A Precision Impedance Analyzer to test the inductance. Furthermore, two groups of micro-planar coils with different wire width, wire distance and wire thickness are fabricated by using electrochemical and MEMS technology.

There are both five coils in the first group and the second group. In the first group, set the turn numbers of the coils 20 , 21, 22, 23 and 24 respectively, the wire width 40um and the distance between wires 40um. In the second group, set the turn numbers of the coils 20, the wire width $40 \mathrm{um}, 45 \mathrm{um}$, 50um, 55um and 60um respectively, the distance between the wires 40um.

In the experiment, we give alternate current in $1 \mathrm{MHz}$ to the two groups of coils, and then test the inductance values. Fig.4 reveals the relationship between the test inductance and theoretical inductance of the first group of coils, where $L^{\prime}$ ' is the theoretical inductance and $L$ " is the test inductance. If the wire width and the distance between the wires remain unchanged, the inductance value will increase with the increasing coil turn numbers. The theoretical result and the test result are consistent, and the deviation between the test inductance and theoretical inductance is small, it is between $3 \%$ and $8 \%$. This is in accordance with the calculation condition of the inductance model of the micro-planar coil.

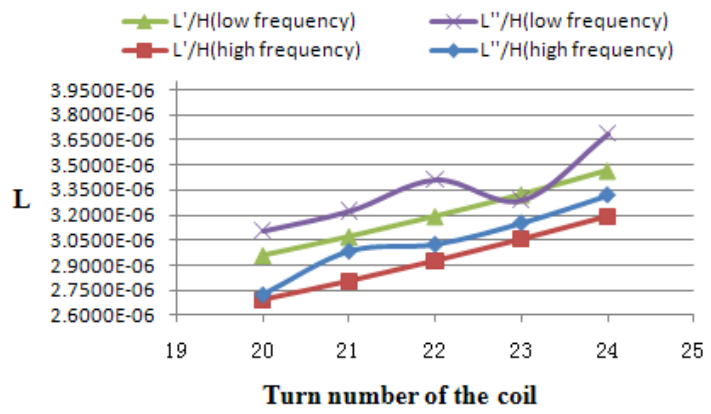

Figure 4. The relationship between the test inductance and theoretical inductance of the first group of coils



Figure 5. The relationship between the test inductance and theoretical inductance of the second group of coils

Fig. 5 reveals the relationship between the test inductance and theoretical inductance of the second group of coils. If the 
coil turn number and the distance between the wires remain constant, the inductance value will decrease with the increasing wire width. Moreover, the curve of the inductance keeps a relatively stable trend, and the deviation between the test inductance and theoretical inductance is between 5\% and $12 \%$. These issues above are all worthy of further research.

\section{B. Experimental test of the impedance model}

When alternating current circulates in the micro-planar coil, alternating magnetic field will generate around the coil. The eddy current effect can also be reflected by the signal of impedance $^{[7]}$. So the impedance is another expression of the inductance.

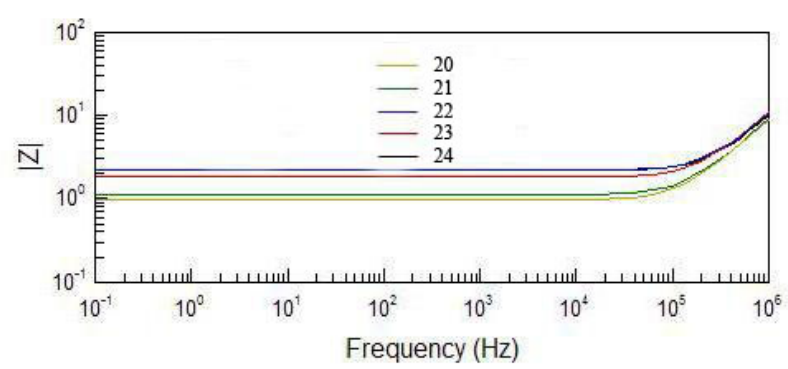

Figure 6. The relationship between impedance and frequency of the first group of coils

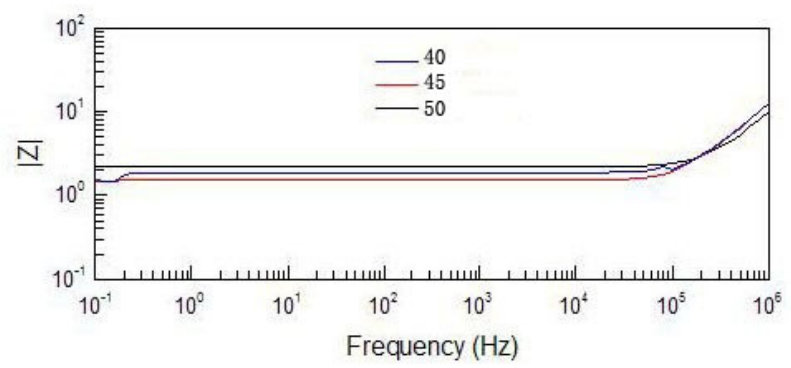

Figure 7. The relationship between impedance and frequency of the second group of coils

In order to verify the impedance model, we use Solartron Electro-chemical impedance system. The two groups of coils which are tested in the previous experiment are also used in this experiment. Give an excitation voltage of $10 \mathrm{mV}$ to the coils, and the frequency incentives from $1 \mathrm{MHz}$ to $0.1 \mathrm{~Hz}$. Then we can get the relationship between the impedance value and the frequency.

Fig.6 and Fig.7 are the relationship between impedance and frequency. The curves of the impedance in Fig.6 and Fig.7 are similar. The impedance starts to be stable when the frequency is about $5 \times 10^{4} \mathrm{MHz}$. When the excitation frequency is between $5 \times 10^{4} \mathrm{MHz}$ and $0.1 \mathrm{~Hz}$, the impedance is extremely stable.

\section{CONCLUSION}

According to the above theoretical analysis and experimental validation of the mathematical model of the micro-planar coil, the inductance and impedance can well describe the near-field eddy current effect of the micro-planar coil. But the inductance model has a more strong computability, and the theoretical result is consistent with the experimental results. The deviation of the inductance between the test results and theoretical results is between $3 \%$ and $12 \%$. So the deviation is in an acceptable range, while it can be detected by Agilent 4294A Precision Impedance Analyzer.

This paper can be a foundation of micro-planar coil for eddy current nondestructive testing. It provides an approach and mathematical foundation to the micro-planar coil for applying in the oil detection depending on the stability and sensitivity of the micro-planar coil.

\section{REFERENCES}

[1] Fan Hongbo, Zhang Yingtang, Ren Guoquan and Chen Fei, "Study on Oil Detection Technology Based on Inductive Wear Debris Sensor”, IEEE ICEMI, vol.2, pp. 810-813, 2009.

[2] John Hansen, Ronald B. Peoples. "Using Eddy Current Testing to Solve Industrial Problems”. Materials Evaluation, vol.5, pp. 543-546, 2006.

[3] Peng Wang, Daoyu Qin, Zhibin Fu. "Study on Signal Detection Technology of Small Inductance Planar Coil”, vol.20, pp.2333-2336, 2007.

[4] Bendong Liu, "Studies on the Theory and Fabrication Process of Micro Electroma-gnetic Relay”, 2008, pp. 18-19.

[5] Yaning Zhu, “Study of Planar spiral inductor in RFIC”, 2006, pp.5.

[6] Bai Song, "A Pulsed Magnetic Field Generation Device based on Power Mosfet”, pp.180, May 2003.

[7] Peng Wang, Tianhuai Ding, Zhibin Fu. "Mathematic model and experimental test for near-filed eddy current of small planar inductance coil”, vol.49, pp.64-67, 2005. 